02

\title{
Проекционный спиновый шум в оптических квантовых датчиках на тепловых атомах
}

\author{
() А.К. Вершовский, ${ }^{1}$ С.П. Дмитриев, ${ }^{1}$ Г.Г. Козлов, ${ }^{2}$ А.С. Пазгалев, ${ }^{1}$ М.В. Петренко ${ }^{1}$ \\ ${ }^{1}$ Физико-технический институт им. А.Ф. Иофрфе РАН, \\ 194021 Санкт-Петербург, Россия \\ 2 Лаборатория оптики спина, \\ Санкт-Петербургский государственный университет, \\ 198504 Санкт-Петербург, Россия \\ e-mail: antver@mail.ioffe.ru
}

Поступило в Редакцию 27 декабря 2019 г.

В окончательной редакции 10 марта 2020 г.

Принято к публикации 10 марта 2020 г.

\begin{abstract}
Теоретически и экспериментально исследованы принципиальные ограничения, накладываемые спиновыми или атомными квантовыми проекционными шумами на чувствительность оптических квантовых датчиков на тепловых атомах (к этому классу устройств относятся стандарты частоты, магнитометры и гироскопы, использующие оптическое детектирование электронного парамагнитного резонанса). Продемонстрирован эффект роста среднеквадратичной амплитуды проекционного шума в магнитометрической схеме под воздействием сильной оптической накачки, предложено и экспериментально подтверждено его объяснение показано, что в широком диапазоне мощностей накачки этот эффект объясняется инвариантностью интегральной мощности проекционного шума по отношению к ширине линии магнитного резонанса. Проведено экспериментальное исследование параметров проекционного шума в магнитометрическом квантовом датчике, даны рекомендации по оптимизации параметров датчика.
\end{abstract}

Ключевые слова: спиновый проекционный шум, оптически детектируемый магнитный резонанс, квантовый датчик, квантовый магнитометр, стандарт частоты, ядерный магнитный гироскоп.

DOI: 10.21883/JTF.2020.08.49533.438-19

\section{Введение}

Благодаря появлению компактных лазерных источников узкополосного оптического излучения, последнее десятилетие характеризуется бурным ростом интереса к оптическим квантовым датчикам или сенсорам, т. е. устройствам, использующим оптическое управление состоянием ансамбля атомов методами электронного парамагнитного резонанса (ЭПР), и оптическое детектирование этого состояния. Несмотря на появление многочисленных новых схем, использующих лазерное охлаждение атомов, все более активно разрабатываются и находят все более широкое применение квантовые датчики, работающие на ансамблях тепловых атомов меньшее время жизни атомных состояний в этих схемах компенсируется их простотой, компактностью, а главное - существенно большим количеством опрашиваемых атомов.

В число таких устройств входят: магнитометры [1] и стандарты частоты [2], использующие переходы ЭПР в зеемановской или сверхтонкой структуре основного состояния щелочных атомов; магнитометры на переходах в метастабильном состоянии гелия-4 [3]; гироскопы, использующие, помимо ЭПР, ядерный магнитный резонанс (ЯМР) в инертных газах, и, по сути, являющиеся комбинацией ЭПР и ЯМР магнитометров, связь между которыми осуществляется посредством спин-обменного взаимодействия между электронными и ядерными парамагнетиками [4,5].

Известно [1], что наиболее фундаментальное ограничение на чувствительность квантовых датчиков определяется количеством опрашиваемых атомов и временем их релаксации. Обусловленный конечностью числа атомов и возможных значений проекций их моментов статистический шум называется спиновым или атомным квантовым проекционным шумом [6]. Исследование этого шума представляет самостоятельный интерес, поскольку позволяет получать информацию о квантовой системе в отсутствие внешних возмущающих воздействий [7]. Проекционный шум в схемах с лазерным охлаждением всесторонне изучен, и предложен ряд схем, позволяющих уменьшить его величину разнообразными методами сжатия квантовых состояний, в том числе в магнитометрических схемах [8-11]. Что касается квантовых датчиков на тепловых атомах, то в течение нескольких десятилетий основным принципиальным фактором, ограничивающим их чувствительность, считался фотонный дробовый шум [12], что также было обусловлено: вопервых, большим количеством атомов в этих схемах, и соответственно относительно низкой величиной их статистических флуктуаций и, во-вторых, недостаточно высокой эффективностью широко использовавшегося метода детектирования сигнала ЭПР по поглощению пробного света. Развитие лазерных методов накачки и 
регистрации магнитного резонанса в сочетании с общей тенденцией миниатюризации квантовых датчиков на тепловых атомах (как для задач навигации, так и для задач медицинской диагностики, а именно магнитоэнцефалографии и магнитокардиографии [13]), однако, привело к относительному снижению вклада фотонного шума, и, как следствие, к необходимости учета квантовых проекционных шумов. Влияние проекционных шумов и возможные способы его снижения посредством квантового неразрушающего измерения (QND - quantum nondemolition measurement) применительно к датчикам на тепловых атомах теоретически рассмотрены в [14-16] и экспериментально исследованы в [17]. Настоящая работа посвящена исследованию проявлений квантового проекционного шума, определяющих предельно допустимый уровень чувствительности в квантовых датчиках, и ее результаты применимы ко всем типам перечисленных выше устройств.

\section{1. Теория}

Рассмотрим ансамбль из $N_{A t}$ атомов $\left(N_{A t}=n V\right.$, где $n$ - концентрация атомов, $V$ - объем), характеризующихся полным угловым моментом $F$, так что максимальное значение любой проекции суммарного момента $M_{F}$ равно $M_{F \max }=F N_{A t}$. Введем понятие спиновой поляризации среды $Q \equiv M_{F} / M_{F \max }(-1 \leq Q \leq 1)$. Вариация поляризации (она же интегральная мощность шума поляризации $P_{I Q}$ ) может быть записана, как

$$
P_{I Q} \equiv\left\langle\Delta^{2} Q\right\rangle=\frac{k_{F}}{N_{A t}},
$$

где $k_{F}$ - коэффициент, связанный с неравномерностью распределения статистических вероятностей реализации возможных проекций суммарного углового момента; для частиц с $F=1 / 2$ (простейшая система с двумя возможными значениями проекции момента) $k_{F}=1$. Для „идеального“ атома, проекция момента которого на любую ось с равной вероятностью может принимать $2 F+1$ значений:

$$
k_{F}=\frac{F+1}{3 F} .
$$

При больших $F$ дискретное распределение переходит в непрерывное распределение Ирвина-Холла (IrwinHall distribution); поскольку значение поляризации определено на интервале от -1 до +1 , при $F \rightarrow \infty$ ее вариация равна $4\left(N_{A t} / 12\right)=N_{A t} / 3$, и соответственно $k_{F}=1 / 3$.

Согласно [18], для атомов с ядерным моментом $I$ и полным моментом $F_{ \pm}=I \pm 1 / 2$ шумы поляризации в основном состоянии должны считаться отдельно для каждого из двух сверхтонких уровней:

$$
k_{F}=n_{+} k_{F+}+n_{-} k_{F-},
$$

где

$$
k_{F \pm}=\frac{F_{ \pm}+1}{3 F_{ \pm}}
$$

a $n_{+}, n_{-}$- относительные населенности уровней $\left(n_{+}+n_{-}=1\right)$. Число подуровней (значений проекции момента) каждого уровня равно $2 F_{ \pm}+1$, общее число подуровней равно $2(2 I+1)$, и в случае равновесной заселенности сверхтонких уровней

$$
n_{ \pm}=\frac{2 F_{ \pm}+1}{2(2 I+1)}
$$

Это соотношение верно при условии равномерной заселенности подуровней (высокотемпературное приближение) и при отсутствии возмущающих факторов, в частности - оптической накачки. При регистрации сигнала оптическими средствами следует также учитывать, что регистрация поляризации двух сверхтонких уровней осуществляется с разной эффективностью, о чем будет более подробно сказано далее.

Для перехода к спектральной мощности шума введем понятие события проекции момента для одного атома: динамика атомного момента обусловлена релаксацией с характерным временем $T_{2}$, и соответственно количество событий проекции момента в $1 \mathrm{~s}$ для атома равно $1 / T_{2}$, а для ансамбля $-N_{A t} / T_{2}$. Соответственно спектр мощности шума атомного ансамбля описывается контуром Лоренца $L(\omega) \equiv 1 /\left[1+(\omega / \Gamma)^{2}\right]$ с полушириной $\Gamma=1 / T_{2}$ и полной мощностью $P_{I Q}$.

В магнитном поле при наблюдении продольной по отношению к полю, а потому неподвижной, компоненты магнитного момента центр шумового контура находится на нулевой частоте. То же верно для поперечной компоненты момента в системе координат, вращающейся с частотой Лармора. Следовательно, в лабораторной системе координат спектр шума переносится из окрестности нулевой частоты в окрестность ларморовской частоты $\omega_{0}$ и регистрируется в виде резонансного контура с полушириной Г. Такая ситуация реализуется в так называемых $M_{X}$ схемах квантовых датчиков $[3,19,20]$, в которых детектируется значение проекции вращающейся поперечной компоненты момента на неподвижный пробный луч. Выражение для спектральной мощности шума поляризации $P_{Q}(\omega)$ в полосе $\Delta \omega=1 \mathrm{~s}^{-1}$ записывается в виде

$$
P_{Q}(\omega)=T_{2}\left\langle\Delta^{2} Q\right\rangle L(\omega)=\frac{k_{F}}{\pi N_{A t}} \frac{\Gamma}{\Gamma^{2}+\left(\omega-\omega_{0}\right)^{2}},
$$

а интегрирование (6) по частоте, как и следовало ожидать, дает величину $P_{I Q}$, выраженную формулой (1). Важный практический вывод из (1) и (6) - интегральная мощность шума поляризации не зависит от ширины линии резонанса.

Формулы (1)-(6) получены в предположении о равномерном распределения значений проекции момента $F_{x}$ на произвольную ось $x$. Такое „одномерное“ представление может показаться недопустимым упрощением: как показано, например, в [21], все основные зависимости для проекционных шумов углового момента могут быть получены моделированием классической вероятности 
случайного проецирования трехмерного момента $F$ на сфере радиусом $r=\sqrt{ }(F(F+1))$. Для проверки применимости приведенных выше формул к динамике трехмерного момента мы построили простую численную модель, в которой динамика атомной поляризации описывалась в рамках статистики двумя способами: $1-$ в „одномерном“ приближении равномерного распределения значений проекции $F_{x}$ момента на произвольную ось $x$, и $2-$ в „трехмерном“ приближении равномерного распределения углового момента по сфере. В модели считалась как вариация распределения (1), так и спектральная мощность квантового шума ансамбля независимых моментов. Результаты моделирования (рис. 1) показали, что в случае „идеального“ атома коэффициент $k_{F}$ хорошо описывается формулой (2) как в одномерном, так и в трехмерном представлении.

Амплитуда проекционного шума, регистрируемого в эксперименте, определяется эффективностью, с которой мы измеряем состояние атомарной среды. Рассмотрим схему, в которой регистрация состояния атома, находящегося в ячейке с буферным газом, осуществляется по повороту азимута поляризации пробного луча (peализация наиболее распространенного варианта такой схемы рассмотрена ниже в разд. 2). В общем случае оптическая линия поглощения среды описывается контуром Фойхта, причем однородное уширение - как естественное $\Gamma_{L}$, так и обусловленное столкновениями с буферным газом $\Gamma_{G}-$ формирует крылья линии (оптические частоты и ширины традиционно выражаются в единицах обычной частоты, т.е. герц). С увеличением отстройки $\Delta v_{F F^{\prime}}=v-v_{F F^{\prime}}$ частоты $v$ детектирующего излучения от центра линии поглощения атома $v_{F F^{\prime}}$ (индексы $F$ соответствуют сверхтонким уровням основного состояния атома, индексы $F^{\prime}-$ уровням возбужденного состояния) поглощение в среде падает пропорционально $\Gamma_{G}^{2} / \Delta v_{F F^{\prime}}^{2}$, а преломление - пропорционально $\Gamma_{G} / \Delta v_{F F^{\prime}}$. Как показано [15], существует оптимальное соотношение между интенсивностью пробного луча и его расстройкой $\Delta v_{F F^{\prime}}$. Если частота $v$ отстроена достаточно далеко (в условиях эксперимента, описанного ниже, на десять и более ширин оптического перехода) от $v_{0}$, то поглощением пробного света можно пренебречь, и считать, что он не изменяет состояние атомарной среды, т.е. реализуется QND - квантовое неразрушающее измерение. В такой схеме количество фотонов, опрашивающих один атом в течение его времени жизни, может быть много большим единицы, и атомный проекционный шум может преобладать над фотонным дробовым.

В простейшей балансной схеме детектирующее излучение после ячейки поступает на анализатор поляризации света, ось которого наклонена на $45^{\circ}$ относительно среднего направления азимута поляризации пробного луча. Полезным сигналом является разность потоков фотонов $\Delta N_{P h}^{\prime}$ в двух выходных каналах анализатора:

$$
\Delta N_{P h}^{\prime}=N_{P h}^{\prime} \sin \left(\frac{\pi}{4}\right) \sin (\Delta \varphi) \approx(1 / \sqrt{2} N)_{P h}^{\prime} \Delta \varphi,
$$

где $\Delta \varphi-$ угол поворота азимута поляризации детектирующего излучения при взаимодействии с атомарной средой, а $N_{P h}^{\prime}-$ суммарный поток фотонов в двух каналах.

Предположим, что изменение поляризации $Q$ на величину $\Delta Q$ вызывает изменение потока фотонов $\Delta N_{P h}^{\prime}$, попадающих за то же время на фотоприемник. Введем величину $k_{D e t}=\Delta N_{P h}^{\prime} / \Delta Q$. Тогда интегральная мощность шума фототока (выраженного в количестве фотоэлектронов в секунду), регистрируемого при детектировании этого света на фотоприемнике, равна

$$
P_{i Q}=k_{P h D}^{2} k_{D e t}^{2} P_{I Q},
$$

где $k_{P h D}-$ эффективность преобразования потока фотонов в фототок. Рассмотрим ячейку длиной $L$ и детектирующий луч поперечным сечением $s=(\pi / 4) d^{2}$ (диаметр луча $d$ не превышает поперечное сечение ячейки, так что $V=s L)$. При постоянной мощности детектирующего излучения полный угол поворота поляризации $\Delta \varphi$ пропорционален концентрации атомов и длине пути луча в поляризованной атомарной среде, но не зависит от сечения луча:

$$
\Delta \varphi=\chi Q L n .
$$

Здесь $\chi\left[\mathrm{rad} \cdot \mathrm{cm}^{2}\right]-$ угол поворота поляризации детектирующего света на единице длины при единичной концентрации атомов и полной поляризации ансамбля $Q=1$. Величина $\chi$ пропорциональна силе осциллятоpa $f_{D 1}$ оптического перехода (для $D_{1}$ линии цезия $\left.f_{D 1} \approx 1 / 3\right)$, и обратно пропорциональна отстройке частоты пробного луча.

Как известно [22,23], в условиях, когда доминирует спин-обмен, распределение населенностей $\rho\left(F, m_{F}\right)$ по магнитным подуровням $m_{F}$ сверхтонких уровней $F$ описывается спиновой температурой $\beta$ [24]: $\rho\left(F, m_{F}\right)=C \exp \left(\beta m_{F}\right)$, где $C-$ нормирующий множитель для всех магнитных подуровней основного состояния; при этом нормированная на единицу электронная спиновая поляризация основного состояния $Q_{S}$ оказывается равной $Q_{S}=\operatorname{th}(\beta / 2)$. В этих условиях коэффициент поворота плоскости поляризации $\chi$ удается выразить [25] не через отдельные населенности магнитных подуровней, а через единственный параметр $Q_{S}$, от которого зависят коэффициенты $A_{F F^{\prime}}\left(Q_{S}\right)$ :

$$
\begin{aligned}
\chi & =-\frac{\pi}{2} r_{0} c f_{D 1} \sum_{F, F^{\prime}} A_{F F^{\prime}}(P) \operatorname{Im}\left[V\left(\Gamma_{G}, \Delta v_{F F^{\prime}}\right)\right] \\
& \approx-\frac{1}{6} r_{0} c \sum_{F, F^{\prime}} \frac{A_{F F^{\prime}}\left(Q_{S}\right)}{\Delta v_{F F^{\prime}}} .
\end{aligned}
$$

Здесь $\quad r_{0}=2.83 \cdot 10^{-13} \mathrm{~cm} \quad-\quad$ классический радиус электрона, $c-$ скорость света, 


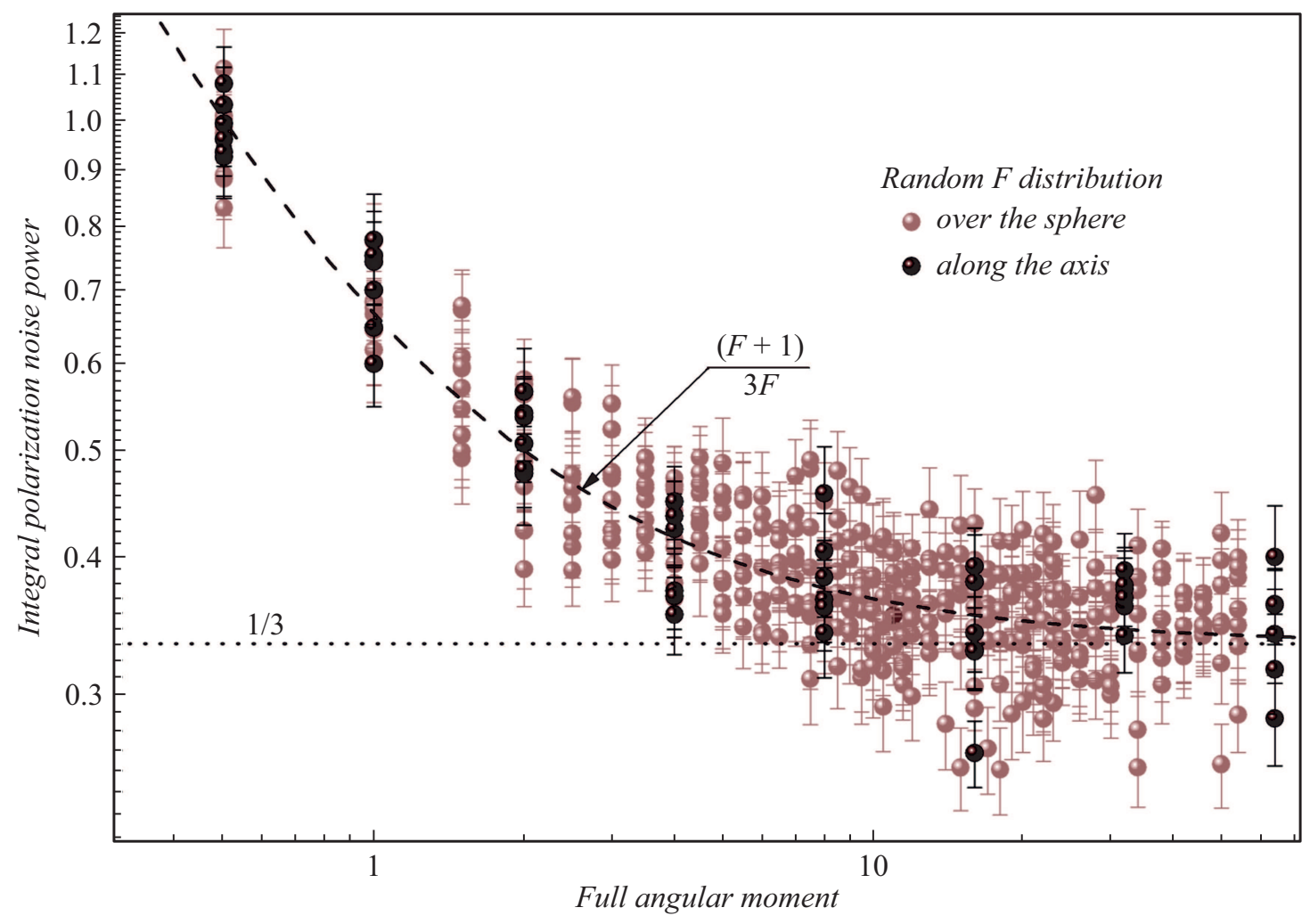

Рис. 1. Результат численного моделирования динамики спиновой поляризации: спектральная мощность квантового шума ансамбля независимых моментов „идеального“ атома с одним сверхтонким уровнем и полным моментом $F$. Темные кружки - приближение равномерного распределения значений проекции момента $F_{x}$ на произвольную ось $x$ („одномерное“), светлые - приближение равномерного распределения углового момента по сфере радиусом $r=\sqrt{ }(F(F+1))$ („трехмерное“).

$\operatorname{Im}\left[V\left(\Gamma_{G}, v-v_{F F^{\prime}}\right)\right] \approx 1 /\left(\pi \Delta v_{F F^{\prime}}\right) \quad$ - мнимая часть контура Фойхта в пределе большой расстройки $\Delta v_{F F^{\prime}} \gg \Gamma_{G}$. Суммирование с весами $A_{F F^{\prime}}\left(Q_{S}\right)$ производится по оптическим переходам между сверхтонкими уровнями основного и возбужденного состояния. Так, для неполяризованного цезия $A_{33}=$ $=7 / 64, \quad A_{34}=-63 / 64, \quad A_{43}=105 / 64, \quad A_{44}=15 / 64$. Зависимость $A_{F F^{\prime}}$ от спиновой поляризации $Q_{S}$ в случае сильного спинового обмена приведена в [25].

Вне приближения спиновой температуры, например, когда скорость оптической накачки превышает темп спиновой релаксации, распределение населенностей не может быть описано единственным параметром спиновой поляризации $Q_{S}$. В этом случае для расчета величины $\chi$ необходимо производить расчет населенностей всех магнитных подуровней $\rho\left(F, m_{F}\right)$, и производить их суммирование с весами $C\left(F, m_{F} \leftrightarrow F, m_{F^{\prime}}\right)$, пропорциональными разности вероятностей переходов $B_{ \pm}\left(F, m_{F} \leftrightarrow F^{\prime}, m_{F^{\prime}}=m F^{\prime}=m^{\prime} \pm 1\right)$, вызванных излучением противоположных циркулярных поляризаций (см., например, [25], выражение (2.96)):

$$
\chi \approx-\frac{1}{6} r_{0} c \sum_{\substack{F, F^{\prime} \\ m_{F}, m_{F^{\prime}}}} \rho\left(F, m_{F}\right) C\left(F, m_{F} \leftrightarrow F^{\prime}, m_{F^{\prime}}\right) \frac{1}{\Delta v_{F F^{\prime}}}
$$

Согласно (7) и (8), и с учетом того, что ток фотоприемника связан с потоком фотонов выражением $I_{P h D}=e k_{P h D} N_{P h}^{\prime}$, полная мощность шума фототока равна

$$
P_{i Q}=\frac{k_{F} I_{P h D}^{2} \chi^{2} L n}{s}
$$

Спектральная мощность шума поперечной компоненты в центре контура Лоренца в полосе $1 \mathrm{~s}^{-1}$ равна

$$
P_{Q}\left(\omega_{0}\right)=\frac{P_{i Q}}{\pi \Gamma}=\frac{k_{F} I_{P h D}^{2} \chi^{2} L n}{\pi \Gamma s},
$$

а величина регулярного (полезного) сигнала $S_{R}$ :

$$
S_{R}=(1 / \sqrt{2}) I_{P h D} Q_{D} \chi L n,
$$

где $Q_{D}-$ проекция спиновой поляризации на направление пробного луча: $Q_{D} \leq Q$. В частности, в $M_{X}$ схемах прецессирующий суммарный момент отклоняется резонансным радиочастотным полем на угол $\Theta$ от направления вектора магнитного поля; при этом, как следует из уравнений Блоха, оптимум чувствительности реализуется при $\Theta=45^{\circ}$, и $Q_{D}=Q \sin \Theta=Q / \sqrt{ } 2$.

Минимальная вариация частоты, детектируемая с помощью квантового датчика, равна спектральной плотности шума в центре резонансной линии, отнесенной 
к крутизне резонанса $S_{R} / \Gamma$. Учтем, помимо проекционного, дробовый шум фототока, характеризующийся спектральной мощностью в полосе $1 \mathrm{~s}^{-1}$ :

$$
P_{i S h}(\omega)=\frac{1}{\pi} e I_{P h D}
$$

Сравнивая (13) с (15), можно вычислить отношение мощности атомного проекционного шума в центре резонансной линии к мощности дробового шума:

$$
k_{Q / S h}=k_{F} \chi^{2} N_{e}^{\prime} T_{2} \frac{L n}{s}=k_{F} \frac{\chi^{2}}{s^{2}} N_{e}^{\prime} T_{2} N_{A t},
$$

где $N_{e}^{\prime}=I_{P h D} / e-$ количество фотоэлектронов, регистрируемых в секунду. С учетом спектральной плотности полного шума в центре контура чувствительность датчика в полосе $1 \mathrm{~s}^{-1}$ равна

$$
\delta \omega=\frac{1}{\sqrt{2 \pi N_{e}^{\prime}}} \frac{\sqrt{1+k_{Q / S h}}}{Q_{D} \chi L N T_{2}} .
$$

Для магнитометрического датчика чувствительность к вариации частоты пересчитывается в магнитометрическую чувствительность по формуле $\delta B=\delta \omega / \gamma$, где $\gamma-$ гиромагнитное отношение.

При $k_{Q / S h}<1$ преобладает дробовый шум, и чувствительность датчика растет линейно с ростом количества атомов и времени релаксации [20] (под ростом чувствительности традиционно понимают снижение величины $\delta \omega)$. Увеличение входящих в (16), (17) параметров $N_{e}^{\prime}, \chi, L, n, T_{2}$ приводит к росту чувствительности и одновременно - к росту проекционных шумов. При $k_{Q / S h}>1$ проекционный шум преобладает, и формула (17) в случае полной поляризации $Q_{D}=1$ приводится к виду

$$
\delta \omega=\sqrt{\frac{k_{F}}{\pi T_{2} N_{A t}}} .
$$

В случае простейшей двухуровневой системы $(F=1 / 2)$ коэффициент $k_{F}$ равен единице, и формула (18) совпадает с приведенной в [1]. При $k_{Q / S h}>1$ чувствительность датчика определяется исключительно величинами $N_{A t}=L s n$ и $T_{2}$, причем зависимость от этих факторов становится корневой. Существенно, что при выполнении условия преобладании проекционных шумов дальнейшее увеличение $N_{e}^{\prime}$ и $\chi$ теряет смысл. Более того, оно нежелательно, поскольку оно неизбежно приводит к увеличению поглощения пробного излучения и, как следствие, к снижению отношения сигнал/шум [15]. Таким образом, при заданных $N_{A t}$ и $T_{2}$ основным критерием оптимизации является выполнение условия равенства $k_{Q / S h} \approx 1$. При достаточно больших значениях расстроек $\Delta v_{F F^{\prime}}$ их можно считать равными для всех оптических переходов: $\Delta v_{F F^{\prime}} \approx \Delta v_{d}$, где $\Delta v_{d}$ - отстройка пробного излучения от центра $D_{1}$ или $D_{2}$ линии. Поскольку $\sum_{F, F^{\prime}} A_{F F^{\prime}}(P)=1$, из (10) следует $\chi \approx-\frac{1}{6} r_{0} c / \Delta v_{d}$. Подставляя это выражение в (16), получаем, что равенство $k_{Q / S h}=1$ обеспечивается при выполнении условия

$$
\frac{\Delta v_{d}^{2}}{N_{e}^{\prime}} \approx \frac{k_{F}}{36}\left(r_{0} c\right)^{2} \frac{T_{2} N_{A t}}{s^{2}},
$$

которое для случая $100 \%$ квантового выхода фотоприемника можно записать в виде

$$
\frac{\Delta v_{d}^{2}}{I_{d}} \approx \frac{k_{F}}{36} \frac{\left(r_{0} c\right)^{2}}{h v_{d}} \frac{T_{2} N_{A t}}{s^{2}}
$$

или

$$
\frac{\Delta v_{d}^{2}}{I_{S d}} \approx \frac{k_{F}}{36} \frac{r_{0}^{2} c}{h} \lambda_{d} T_{2} n L,
$$

где $h$ - постоянная Планка, $v_{d}$ и $v_{d}$ - частота и длина волны пробного излучения, $I_{d}-$ его мощность, измеренная на фотодетекторе, а $I_{S d}=I_{d} / s-$ его интенсивность. Выражения (19)-(21) определяют требования к расстройке и интенсивности пробного излучения, обеспечивающим оптимальный уровень его поглощения.

Если релаксация преимущественно определяется спин-обменом (как предполагалось при выводе (10)), то $1 / T_{2}=\Gamma_{S E}=\sigma_{S E} v_{\mathrm{Re} l} n$, где $\sigma_{S E}-$ сечение спинобмена, для Cs, равное $1.4 \cdot 10^{-14} \mathrm{~cm}^{2}$, а $v_{\operatorname{Re} l}=4 \sqrt{\frac{R T}{\pi m}}$ относительная скорость сталкивающихся атомов. Тогда из (21) следует, что

$$
\frac{\Delta v_{d}^{2}}{I_{S d}} \approx \frac{k_{F}}{144} \frac{r_{0}^{2} c}{h} \sqrt{\frac{\pi m}{R T}} \frac{L \lambda_{d}}{\sigma_{S E}} .
$$

Таким образом, в приближении доминирования спинобменной релаксации оптимальная расстройка $\Delta v_{d}$ очень слабо зависит от температуры $T$ и не зависит от концентрации атомов: $\Delta v_{d}=$ const $\cdot I_{S d}^{1 / 2} T^{-1 / 4} L^{1 / 2}$ (для Cs const $=12.25 \mathrm{GHz} \cdot \mathrm{cm}^{1 / 2} \cdot \mathrm{mW}^{-1 / 2} \cdot \mathrm{K}^{1 / 4}$, что хорошо согласуется с экспериментом; соответствующие зависимости приведены на рис. 2, $a$ ).

Этот вывод совершенно нетривиален, поскольку с ростом температуры быстро растет концентрация атомов, а следовательно, - поглощение пробного луча и максимальная амплитуда сигнала достигается при все большей расстройке. Однако оптимизация по максимуму чувствительности приводит к совершенно другому результату. Впрочем, случай чистого спин-обменного уширения (22) достаточно редко реализуется на практике. Самое существенное ограничение применимости формул (19)-(22) связано с уширением резонансной линии как пробным светом, так и светом накачки: с увеличением концентрации атомов возрастает поглощение света (что при постоянной мощности $I_{d}$ на фотоприемнике означает увеличение мощности пробного света, входящего в рабочую ячейку), и уширение светом начинает конкурировать со спин-обменным уширением (далее мы рассмотрим и обратный эффект - сужение линии светом сильной накачки). Поэтому приближение „чистого“ спин-обменного уширения требует не только 

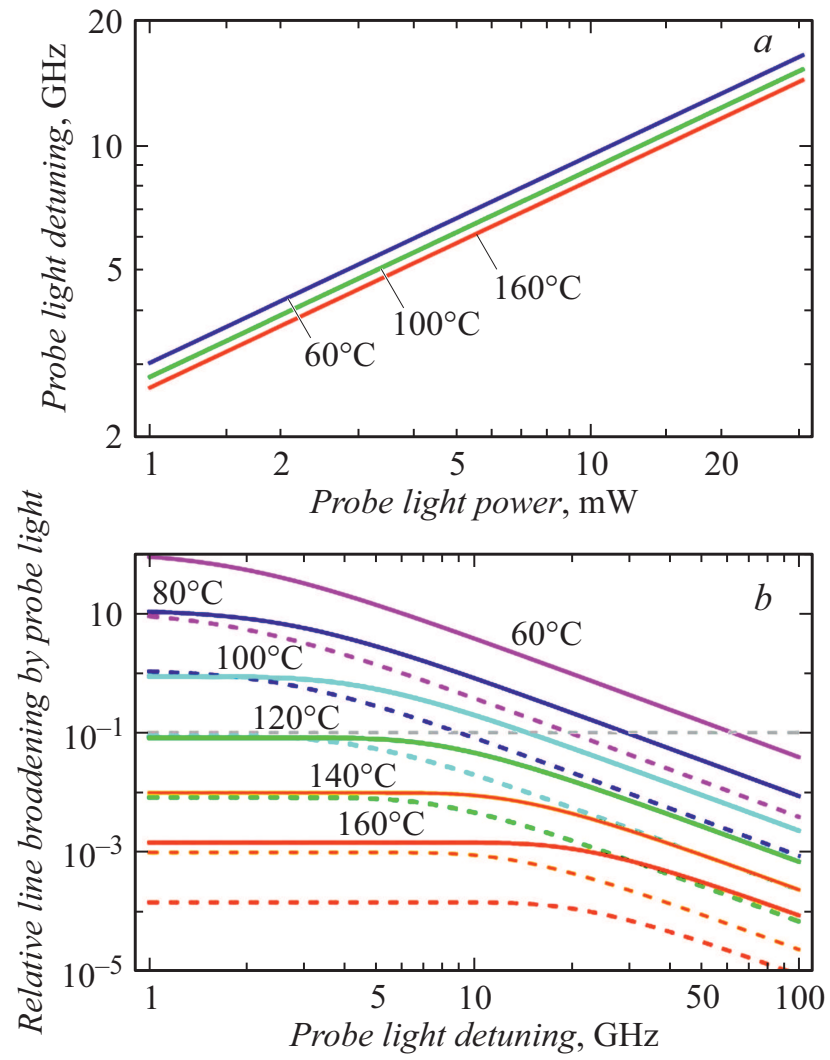

Рис. 2. Теоретические зависимости оптимальных параметров пробного излучения от мощности пробного излучения для датчика на кубической ячейке $L=1 \mathrm{~cm}$ с парами Cs в атмосфеpe 200 Torr $\mathrm{N}_{2}$ при различных температурах: $a-$ оптимальной расстройки пробного излучения от мощности пробного излучения в приближении спин-обменной релаксации без учета уширения излучением; $b-$ уширения пробным излучением, отнесенного к спин-обменной ширине, от расстройки пробного излучения для того же датчика при различных температурах и интенсивности $1 \mathrm{~mW} / \mathrm{cm}^{2}$ (пунктир) и $10 \mathrm{~mW} / \mathrm{cm}^{2}$ (сплошная линия). Горизонтальная серая пунктирная линия - уровень относительного светового уширения $\Gamma_{d} / \Gamma_{S E}=0.1$.

слабой накачки, но и слабого или сильно нерезонансного пробного света: $\Gamma_{d} / \Gamma_{S E} \ll 1$, или

$$
\frac{\Gamma_{d 0} \Gamma_{G}^{2}}{\Delta v_{d}^{2}+\Gamma_{G}^{2}} \ll \sigma_{S E} v_{\operatorname{Re} l} n,
$$

где $\Gamma_{d 0}$ - уширение пробным светом в центре однородного оптического контура, причем $\Gamma_{d 0}$ пропорционально $I_{S d}$ и сложным образом зависит от $L$ и $n$. Пример расчета безразмерного (отнесенного к спинобменному) уширения $\Gamma_{d} / \Gamma_{S E}$ для конкретной системы в приближении экспоненциального (по закону Бугера) поглощения пробного света приведен на рис. $2, b$. В случае невыполнения условия (23) в (19)-(22) необходимо учесть зависимость $T_{2}\left(I_{S d}\right)$.

Итак, оптимизация параметров датчика подразумевает в первую очередь равенство уровней дробовых и проекционных шумов, отношение которых определяется формулой (16). Требуемое равенство обеспечивается при выполнении условия (19)-(21), связывающего параметры регистрируемого пробного излучения (отстройку и силу фототока на фотоприемнике) с параметрами используемой ячейки.

\section{2. Эксперимент}

Параметры проекционных шумов нами были исследованы экспериментально в двухлучевой $\mathrm{M}_{x}$ схеме с детектированием поворота поляризации пробного луча (рис. 3). В ходе эксперимента был обнаружен и исследован эффект увеличения амплитуды проекционного шума при включении сильной оптической накачки, и показано, что он вызван физическими причинами принципиального характера.

Магнитный резонанс детектировался в условиях частичного подавления спин-обменного уширения сильной лазерной накачкой $[13,26,27]$. В отличие от широко известного эффекта SERF [28] это подавление реализуется в ненулевых магнитных полях; оно возникает при высокой степени поляризации атомного ансамбля, когда значительная часть сталкивающихся атомов находится в одном состоянии $m_{F}=F$ (или $m_{F}=-F$ ), и взаимодействие между ними происходит без изменения спинового состояния. В этих условиях нами был зарегистрирован значительный рост шумов на частоте резонанса при включении накачки. Чтобы исключить шумы технического происхождения, в первую очередь обусловленные флуктуациями лазерного излучения, мы использовали регистрацию шумовых и регулярных сигналов одновременно в двух ячейках; луч накачки, так же как и луч детектирования, расщеплялся на два

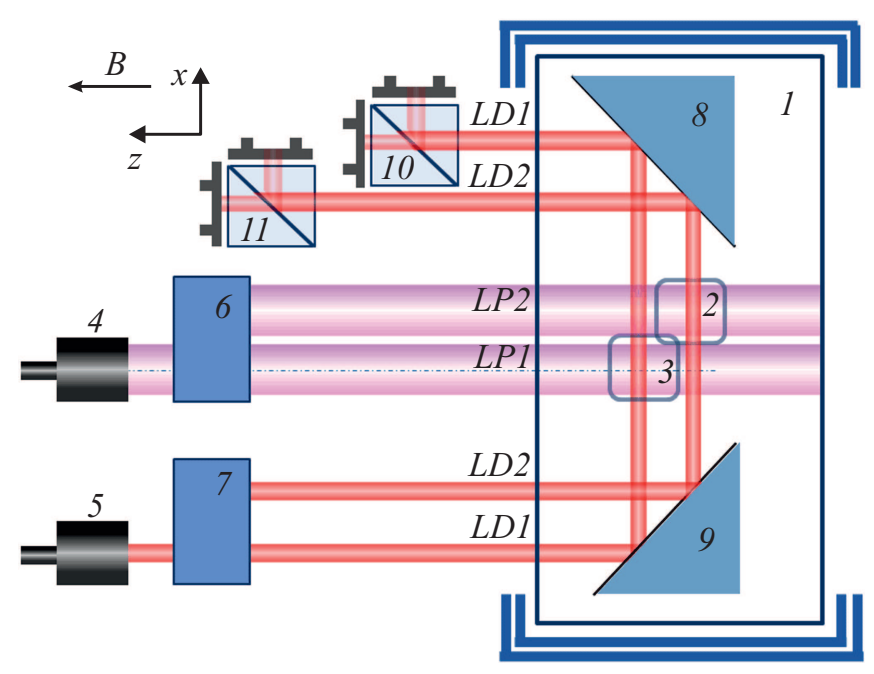

Рис. 3. Упрощенная схема экспериментальной установки: 1 - магнитный экран с термостатом, 2,3 - ячейки с Cs, 4 - ввод излучения накачки, 5 - ввод пробного излучения, 6,7 - оптические расщепители лучей, $8,9-$ зеркала, 10,11 - балансные фотоприемники, $L P 1, L P 2$ - лучи оптической накачки, $L D 1, L D 2$ - пробные лучи. 


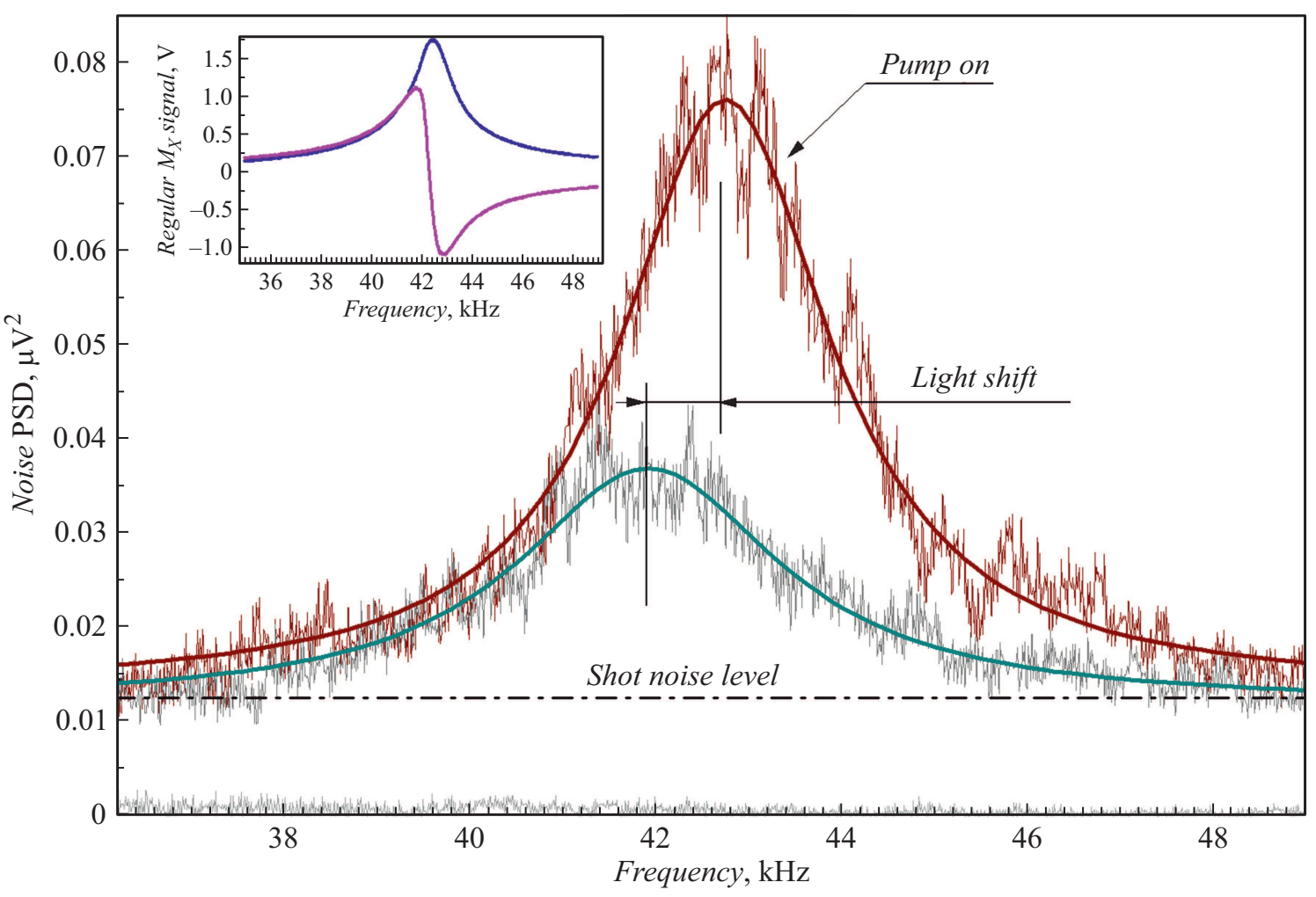

Рис. 4. Пример записи дифференциальных сигналов спектральной мощности шума и их аппроксимаций контурами Лоренца: светлая (голубая в online версии) линия - без оптической накачки, темная (красная в online версии) линия - с оптической накачкой; мощность пробного света $I_{d}=3.5 \mathrm{~mW}$, мощность света накачки $I_{p}=4.0 \mathrm{~mW}$. Сдвиг второго шумового контура обусловлен световым сдвигом частоты резонанса. Штрихпунктирная линия - уровень дробовых шумов фототока. Вставка: регулярный сигнал магнитного резонанса — модуль и Х-компонента.

луча с одинаковыми поляризациями и геометрическими характеристиками, и производилась тщательная балансировка их интенсивностей с точностью порядка единиц процентов. Одновременно с сигналами с двух балансных фотоприемников записывалась и их разность.

Магнитный резонанс возбуждался в двух ячейках размером $8 \times 8 \times 8 \mathrm{~mm}$, содержащих насыщенные пары Cs и азот $\left(\mathrm{N}_{2}\right)$ под давлением 100 Torr при температуре $115^{\circ} \mathrm{C}$. В качестве источников накачки и пробного излучения использовались полупроводниковые лазеры с внешними резонаторами производства компании VitaWave; частота лазера накачки перестраивалась в окрестности перехода $F=3 \leftrightarrow F^{\prime}=3,4 D_{1}$ линии Cs (за точку отсчета была принята частота перехода $F=3 \leftrightarrow F^{\prime}=3 D_{1}$ линии Cs в опорной вакуумной ячейке с парами цезия), частота пробного (детектирующего) лазера была отстроена на $\sim 40 \mathrm{GHz}$ вниз от перехода $F=4 \leftrightarrow F^{\prime}=3 D_{1}$ линии $\mathrm{Cs}$, что при полуширине оптического контура поглощения $\Gamma_{\mathrm{G}} \sim 1 \mathrm{GHz}$ обеспечивало практически полное отсутствие поглощения пробного света, а также близкие с точностью до $25 \%$ коэффициенты эффективности регистрации сигналов на сверхтонких уровнях $F=3$ и $F=4$ основного состояния Cs. Ячейки были расположены на расстоянии $1 \mathrm{~mm}$ одна от другой в многослойном магнитном экране, индукция поля в котором измерялась независимым рубидиевым магнитометром и поддерживалась на уровне $12.0 \mu \mathrm{T}$ при помощи схемы активной стабилизации (на схеме не показаны). Диаметр лучей накачки ( $7 \mathrm{~mm})$ соответствовал размеру ячейки, диаметр лучей детектирования составлял $2.0 \mathrm{~mm}$; оценка величины уширения магнитного резонанса светом накачки в такой конфигурации дает величину порядка $1 \mathrm{kHz} / \mathrm{mW}$ для сверхтонкого уровня $F=3$, непосредственно взаимодействующего со светом накачки. Балансный фотоприемник представлял собой поляризационный расщепитель Thorlabs PBS122 с двумя фотодиодами в общей оправе; коэффициент преобразования мощности регистрируемого излучения $I_{d}$ в фототок $I_{P h D}$ на длине волны $D_{1}$ линии Cs составлял примерно $0.7 \mathrm{~A} / \mathrm{W}$. Преобразование фототока в выходное напряжение (в единицах которого далее приведены результаты эксперимента) осуществлялся трансимпедансным усилителем с коэффициентом преобразования $5.0 \mathrm{~V} / \mathrm{mA}$. Амплитуды регулярного и шумового сигналов в окрестности ларморовской частоты измерялись синхронным детектором SRS SR830, на опорный вход которого подавалась частота с генератора, включенного в режим сканирования.

Сравнение мощностей шумов магнитного резонанса в каждой ячейке с мощностью дифференциального шума 

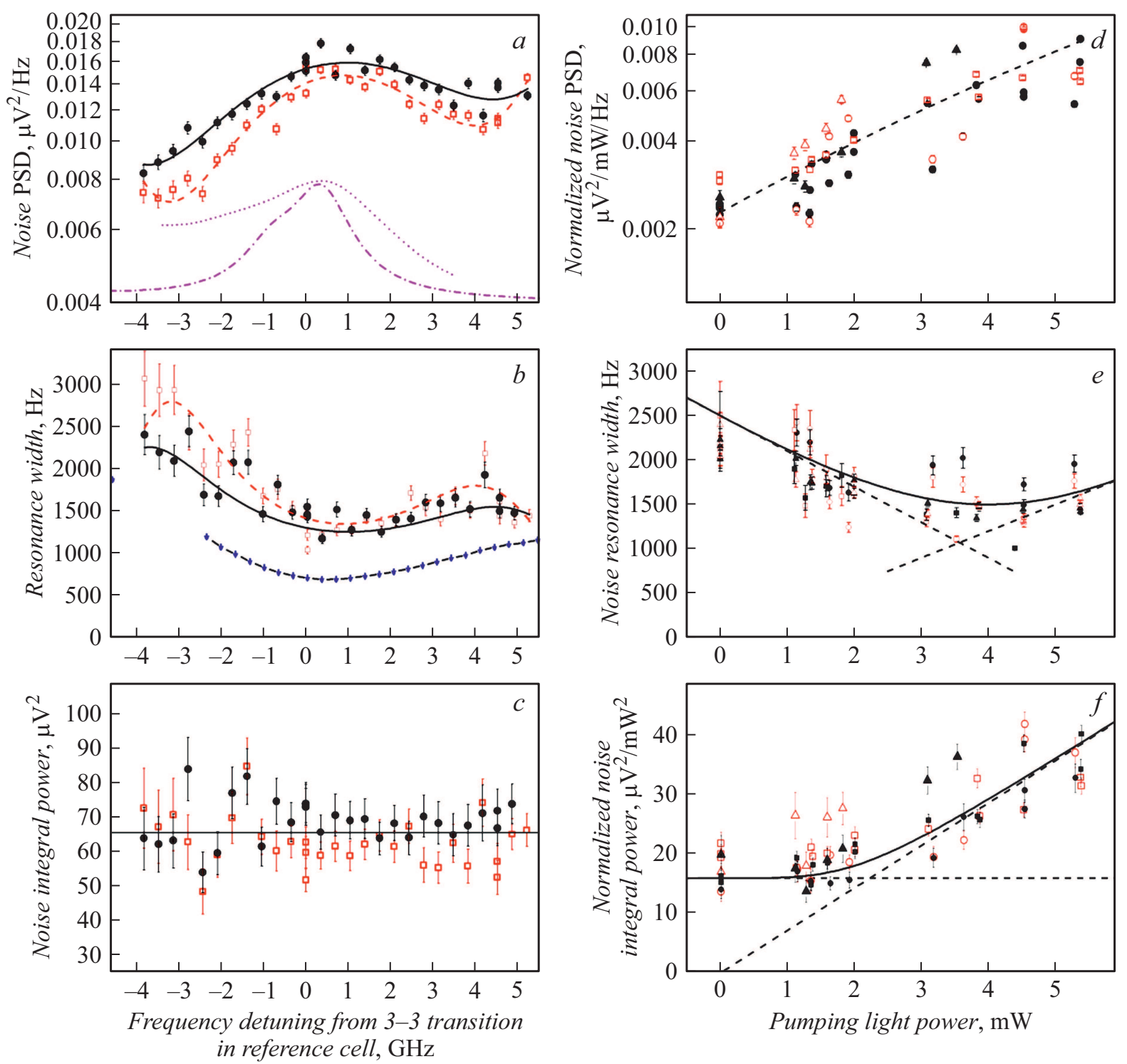

Рис. 5. Параметры шумовых резонансов в двух ячейках с $\mathrm{Cs}+\mathrm{N}_{2}$. Символы с заполнением (черные) - параметры шума в ячейке \#1, без заполнения (красные в online версии) - параметры дифференциального шума, причем мощность шума поделена на 2. Сплошные и пунктирные линии - аппроксимация шумовых зависимостей. $a-c-$ зависимости от отстройки частоты излучения накачки от перехода $F=3 \leftrightarrow F^{\prime}=3 D_{1}$ линии Cs, мощность пробного света $I_{d}=2.5 \mathrm{~mW} . a-$ спектральная плотность мощности шума, дополнительно: точечный пунктир (фиолетовый в online версии) - расчетный оптический контур поглощения, штрих-пунктир (фиолетовый в online версии) - реальный контур поглощения с учетом просветления среды; $b$ - ширины шумовых резонансов, дополнительно: „звездочки“ - ширины регулярных резонансов; $c$ - интегральная мощность шума; сплошная горизонтальная линия - мощность при выключенной накачке. $d-f$ - зависимости от мощности накачки $I_{p}, \triangle: I_{d}=1.14 \mathrm{~mW}, \circ$ : $I_{d}=2.50 \mathrm{~mW}, \square: I_{d}=3.50 \mathrm{~mW} . d-$ нормированная на $I_{d}^{2}$ спектральная плотность мощности шума, $e-$ ширины шумовых резонансов, $f-$ нормированная на $I_{d}^{2}$ интегральная мощность шума.

позволило сделать вывод об отсутствии корреляции шумов резонанса в двух ячейках и соответственно об отсутствии вклада флуктуаций лазерного излучения.

Пример регистрации проекционных шумов приведен на рис. 4; видно, что при включении интенсивной накачки $\left(I_{p}=4.0 \mathrm{~mW}\right)$ частота шумового контура смещается вследствие светового сдвига частоты магнитного резонанса, а мощность в его центре резко возрастает и достигает величины, превышающей мощность дробового шума почти на порядок, что соответствует падению чувствительности датчика примерно в 3 раза по сравнению с оцененной по уровню дробового шума.

Результаты измерений приведены на рис. 5; на левой части рисунка показаны зависимости параметров шумовых и регулярных сигналов в зависимости от отстройки частоты лазера накачки от контура оптического поглощения Cs (точнее - от частоты перехода $F=3 \leftrightarrow F^{\prime}=3 \mathrm{Cs}$ в опорной вакуумной ячейке), на 
правой части - в зависимости от мощности накачки $I_{p}$. На графиках верхнего ряда (рис. 5, a,d) приведены спектральные плотности мощности шума (PSD), на графиках второго ряда (рис. 5, b,e) - полуширины (HWHM) резонансных контуров, на графиках нижнего ряда (рис. $5, b, e)$ - интегральные мощности шума при включенной и выключенной $\left(I_{p}=0\right)$ накачке. Значения мощности дифференциального шума всюду приведены к шкале мощности шума в отдельной ячейке (умножены на коэффициент 1/2). В правой части рисунка приведены измерения, сделанные при трех значениях мощности пробного света; для удобства сравнения значения мощности шума в соответствии с (12), (13) нормированы на $I_{d}^{2}$. Погрешности, обозначенные на рис. 5 вертикальными линиями (error bars), вычислены при аппроксимации записей спектра мощности шума контурами Лоренца, и отражают только статистический разброс данных, но не систематические ошибки.

\section{3. Обсуждение}

Графики рис. 5, $a-c$ подтверждают сделанные ранее выводы: при включении оптической накачки и настройке ее частоты на центр контура оптического поглощения интегральная мощность шума не меняется, но уменьшение ширины резонанса приводит к росту амплитуды проекционного шума. Эти же выводы при мощности накачки $I_{p} \leq 2 \mathrm{~mW}$ подтверждаются графиками рис. $5, d-f$.

При $I_{p}>2 \mathrm{~mW}$, однако, начинается рост интегральной мощности шума, природу которого еще предстоит объяснить; приведенная на рис. 4 запись шума, сделанная при мощности света накачки $I_{p}=4.0 \mathrm{~mW}$, иллюстрирует именно этот случай. Поскольку в области больших мощностей накачки сохраняется отношение мощностей шумов магнитного резонанса в каждой ячейке и мощностей дифференциального шума, вывод об отсутствии корреляции шумов в двух ячейках остается в силе; соответственно у нас есть основания приписать этот рост физическим, а не техническим причинам.

Частичное объяснение роста шумов можно дать, принимая во внимание то факт, что с ростом оптической накачки (по схеме $F=3 \rightarrow F^{\prime}=3, F^{\prime}=4$ ) происходит опустошение нижнего уровня $F=3$ и дополнительное заселение уровня $F=4$ основного состояния, эффективность детектирования для которого почти вдвое выше (для полностью поляризованной среды). Так, для газовых ячеек, использованных в нашем эксперименте, столкновительное уширение оптической линии поглощения примерно равно сверхтонкому расщеплению возбужденного состояния $P_{1 / 2}$, и в соответствии с (10) мы можем грубо оценить эффективности детектирования состояний уровней $F=3$ и $F=4: A_{3}=A_{33}+A_{34}=-56 / 64$, $A_{4}=A_{43}+A_{44}=120 / 64$, и $A_{4} / A_{3}=2.14$. Далее детектирование состояния $F=4$ происходит с несколько большей эффективностью в силу меньшей (на 1/4) расстройки детектирующего лазера. Таким образом, при полной сверхтонкой накачке на уровень $F=4$ можно ожидать увеличения эффективности детектирования момента примерно в 1.4 раза по сравнению с детектированием равновесного состояния, и соответственного увеличения мощности детектируемого шума примерно в 2 раза.

Не исключена также возможность того, что интегральный шум поперечной компоненты возрастает с ростом накачки вследствие квантового принципа неопределенности, накладывающего ограничения снизу на произведение неопределенностей пространственных компонент углового момента [21]: сильная оптическая накачка приводит к уменьшению неопределенности продольной компоненты момента, и, следовательно, к увеличению неопределенностей поперечных компонент (существует, однако, теория, согласно которой сильная накачка должна приводить к противоположному эффекту [29]).

Впрочем, для задачи оптимизации параметров квантового датчика эффект роста интегральных шумов не столь существенен, поскольку он наблюдается при значениях мощности накачки настолько высоких, что подавление спин-обменного уширения уступает место световому уширению резонанса (рис. 4, 5,e), а потому использование таких мощностей не имеет смысла даже без учета избыточных шумов.

Из рис. $5, b$ следует, что ширина шумового резонанса на $500-600 \mathrm{~Hz}$ превышает ширину регулярного сигнала; дополнительное „пролетное“ уширение обусловлено диффузионным движением атомов в пробном луче, приводящим к ограничению времени взаимодействия атома с лучом временами порядка $0.3 \mathrm{~ms}$. Сигнал регулярного резонанса не подвержен пролетному уширению, поскольку фаза прецессии участвующих в нем атомов задана внешним резонансным радиочастотным полем, а потому атомы покидают луч и входят в него в одной и той же фазе. Следовательно, можно ожидать, что совместное воздействие накачки и радиочастотного поля приведет к подавлению пролетного уширения и для шумового контура; а это в свою очередь приведет к дополнительному увеличению амплитуды шумового контура и к дополнительному $(\sim \sqrt{ } \Gamma)$ снижению чувствительности датчика.

Проверим соответствие полученных экспериментальных результатов выражениям (12), (14), из которых следует, что отношение сигнала к корню из интегральной мощности шума равно

$$
\frac{S}{\sqrt{P_{i Q}}}=Q_{D} \sqrt{\frac{n V}{k_{F}}} .
$$

Наиболее трудно измеряемой в эксперименте величиной в (24) является степень поляризации атомного ансамбля - ее теоретический расчет для схемы накачки, использованной в настоящей работе, был приведен в [30], но для ее экспериментального измерения необходимо знание ряда констант и параметров эксперимента. 
Формула (24) позволяет вычислить степень поляризации напрямую, зная параметры регулярного и шумового сигналов и число опрашиваемых атомов.

Для сравнения эксперимента с теорией возьмем значения шума и сигнала, приведенные на рис. 4, и полученные при следующих условиях: $F=4$ (цезий), $k_{F}=0.429, I_{p}=4 \mathrm{~mW}, I_{d}=3.5 \mathrm{~mW}, T=115^{\circ} \mathrm{C}, n=$ $=3.5 \cdot 10^{13} \mathrm{~cm}^{-3}, L=0.8 \mathrm{~cm}, d=0.2 \mathrm{~cm}\left(V=0.025 \mathrm{~cm}^{3}\right.$, $\left.(n V)^{1 / 2}=9.35 \cdot 10^{5},\left(n V / k_{F}\right)^{1 / 2}=1.42 \cdot 10^{6}\right)$, коэффициент преобразования фототока в напряжение $R_{L}=5 \mathrm{~V} / \mathrm{mA}$, амплитуда $M_{X}$ сигнала при оптимальной мощности радиочастотного поля $S_{U}=(1.51 \pm 0.02) \mathrm{V}$, или $S=$ $=3.02 \cdot 10^{-4} \mathrm{~A}$, интегральная мощность шума $P_{u Q}=(132 \pm 8) \mu \mathrm{V}^{2}$, или $P_{i Q}=5.28 \cdot 10^{-18} \mathrm{~A}^{2}$. Следовательно, в нашем эксперименте $S / \sqrt{ } P_{i Q}=1.36 \cdot 10^{5}$.

Согласно (20), проекция поляризации на пробный луч равна $Q_{D}=0.095$, а полная (с учетом угла $\Theta=45^{\circ}$ ) поляризация $Q=0.134$.

Сравнивая этот результат с [30] и сопоставляя его с величиной спин-обменного сужения линии, можно заключить, что полученное нами, согласно (24), значение поляризации занижено примерно в 2 раза. Это может быть объяснено неточностью определения количества атомов в объеме - двукратная ошибка в определении концентрации насыщенного пара соответствует ошибке в определении эффективной температуры ячейки, т.е. температуры наиболее холодного ее участка, всего на несколько градуса. Впрочем, для шумовых экспериментов такое расхождение с оценочными формулами следует признать вполне удовлетворительным.

\section{Выводы}

Задача разработки и оптимизации современных схем квантовых датчиков на тепловых атомах, использующих миниатюрные ячейки и современные высокоэффективные методы регистрации сигнала магнитного резонанса, требует учета квантовых проекционных шумов. В работе исследованы проекционные шумы в схеме квантовых оптических датчиков, использующих квантовое неразрушающее измерение, и выведены выражения, позволяющие оптимизировать режимы датчика по критерию чувствительности, обеспечив равенство спектральных мощностей проекционного и дробового шума в центре линии магнитного резонанса. Обнаружен и исследован эффект роста спектральной мощности проекционного шума в магнитометрической схеме при включении сильной оптической накачки, приводящий к существенному снижению чувствительности квантового датчика; показано, что он объясняется инвариантностью интегральной мощности проекционного шума по отношению к ширине линии магнитного резонанса. Также при дальнейшем увеличении мощности накачки обнаружен эффект роста интегральной мощности проекционного шума, требующий дополнительного исследования. Даны рекомендации по оптимизации параметров датчика. Результаты работы применимы ко всем типам квантовых датчиков на тепловых атомах - магнитометрам, гироскопам, стандартам частоты.

\section{Финансирование работы}

Теоретическая часть исследования (раздел статьи „Теория“ $)$ выполнена при финансовой поддержке РФФИ в рамках научного проекта 19-52-12054. Экспериментальная часть исследования (раздел статьи „Эксперимент“") выполнена при финансовой поддержке РФФИ в рамках научного проекта № 19-29-10004.

\section{Конфликт интересов}

Авторы заявляют, что у них нет конфликта интересов.

\section{Список литературы}

[1] Budker D., Romalis M. // Nature Phys. 2007. Vol. 3. P. $227-$ 234. DOI: $10.1038 /$ nphys566

[2] Vanier J. et al. // IEEE Trans. Instrum. Meas. 2003. Vol. 52. N 3. P. $822-831$. DOI: $10.1109 /$ TIM.2003.814687

[3] Александров Е.Б., Вершовский А.К. // УФН. 2009. Т. 179. Вып. 6. C. 605-637. DOI: 10.3367/UFNr.0179.200906f.0605 [Aleksandrov E.B., Vershovskii A.K. // Phys. Uspekhi. 2009. Vol. 52. N 6. P. 573-601.] DOI: $10.3367 /$ UFNe.0179.200906f.0605

[4] Walker T., Larsen M. // Adv. Mol. Opt. Phys. 2016. Vol. 65. P. 373-401. DOI: 10.1016/bs.aamop.2016.04.002

[5] Vershovskii A.K. et al. // Gyroscopy Navig. 2018. Vol. 9. N 3. P. 162-176. DOI: 10.1134/S2075108718030100

[6] Itano W.M. et al. // Phys. Rev. A. 1995. Vol. 47. N 5. P. 3554 3570. DOI: 10.1103/PhysRevA.47.3554

[7] Zapasskii V.S. // Adv. Opt. Photon. 2013. Vol. 5. N 2. P. 131168. DOI: 10.1364/AOP.5.000131

[8] Wineland D.J. et al. // Phys. Rev. A. 1994. Vol. 50. N 1. P. 67-88. DOI: 10.1103/PhysRevA.50.67

[9] Geremia J., Stockton J.K., Mabuchi H. // Phys. Rev. Lett. 2005. Vol. 94. N 20. P. 203002. DOI: 10.1103/PhysRevLett.94.203002

[10] Sewell R.J. et al. // Phys. Rev. Lett. 2012. Vol. 109. N 25. P. 253605. DOI: 10.1103/PhysRevLett.109.253605

[11] Hosten O. et al. // Nature. 2016. Vol. 529. N 7587. P. 505-508. DOI: $10.1038 /$ nature 16176

[12] Александров и др. // Опт. и спектр. 1995. Т. 78. № 2. C. 325-332. [Aleksandrov E.B. et al. // Opt. spectr. 1995. Vol. 78. P. 292-298.]

[13] Ossadtchi A.E. et al. // IEEE Proceedings. International Conference on Laser Optics (ICLO 2018). St.Petersburg, 2018. P. 543-543.

[14] Geremia J. et al. // Phys. Rev. Lett. 2003. Vol. 91. N 25. P. 250801. DOI: 10.1103/PhysRevLett.91.250801

[15] Auzinsh M. et al. // Phys. Rev. Lett. 2004. Vol. 93. N 17. P. 173002. DOI: 10.1103/PhysRevLett.93.173002

[16] Dong H.-F. et al. // Chin. Phys. Lett. 2019. Vol. 36. N 2. P. 020701. DOI: $10.1088 / 0256-307 X / 36 / 2 / 020701$

[17] Wasilewski W. et al. // Phys. Rev. Lett. 2010. Vol. 104. N 13. P. 133601. DOI: 10.1103/PhysRevLett.104.133601 
[18] Shah V., Vasilakis G., Romalis M.V. // Phys. Rev. Lett. 2010. Vol. 104. N 1. P. 013601.

DOI: 10.1103/PhysRevLett.104.013601

[19] Aleksandrov E.B., Vershovskiy A.K. In „Optical magnetometry“ / Ed. by D. Budker, D.F.J. Kimball. Cambridge: Cambridge University Press, 2013. P. 60-84.

[20] Вершовский А.К., Пазгалев А.С. // ЖТФ. 2008. Т. 78. Вып. 5. C. 116-124. [Vershovskii A.K., Pazgalev A.S. // Tech. Phys. 2008. Vol. 53. N 5. P. 646-654. https://doi.org/10.1134/S1063784208050198]

[21] Dammeier L., Schwonnek R., Werner R.F. // New J. Phys. 2015. Vol. 17. N 9. P. 093046.

[22] Happer W. // Rev. Mod. Phys. 1972. Vol. 44. N 2. P. 169-249. DOI: https://doi.org/10.1103/RevModPhys.44.169

[23] Будкер Д., Кимбелл Д., Де Милль Д. Атомная физика. Освоение через задачи / Пер. с англ. под ред. Е.Б. Александрова. М.: Физматлит, 2009. 400 с. [Budker D., Kimball D.F., DeMille D.P. An Exploration Through Problems and Solutions. Oxford University Press, 2003.]

[24] Anderson L.W. et al. // Phys. Rev. 1960. Vol. 120. N 4. P. 1279-1289. DOI: https://doi.org/10.1103/PhysRev.120.1279

[25] Seltzer S.J. Developments in Alkali-Metal Atomic Magnetometry / Ph.D. Thesis. Princeton University Princeton, NJ, USA, 2008. $312 \mathrm{p}$.

[26] Bhaskar N.D. et al. // Phys. Rev. A. 1981. Vol. 23. N 6. P. 3048-3064. DOI: https://doi.org/10.1103/PhysRevA.23.3048

[27] Scholtes T. et al. // Phys. Rev. A. 2011. Vol. 84. N 4. P. 043416. DOI: $10.1103 /$ PhysRevA.84.043416

[28] Allred J.C. et al. // Phys. Rev. Lett. 2002. Vol. 89. N 13. P. 130801. DOI: 10.1103/PhysRevLett.89.130801

[29] Lee S.-K. // https://arxiv.org/abs/0804.3585. 2008.

[30] Попов Е.Н. и др. // Письма в ЖЭТФ. 2018. Т. 108. Вып. 8. C. 543-548. DOI: 10.1134/S0370274X18200043 [Popov E.N. et al. // JETP Lett. 2018. Vol. 108. N 8. P. 513-518. DOI: $10.1134 / \mathrm{S} 0021364018200122]$ 